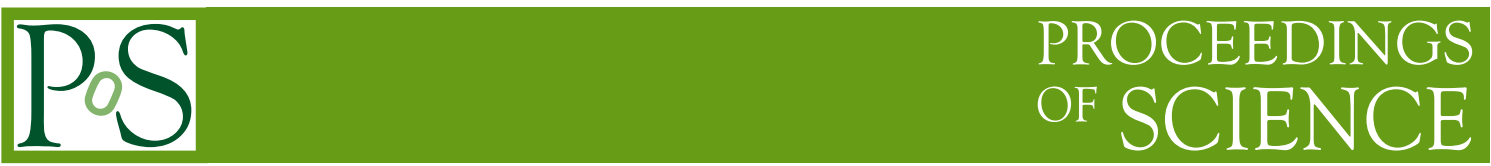

\title{
Measurements of photon-photon fusion at ATLAS
}

\author{
Mateusz Dyndal, on behalf of the ATLAS Collaboration ${ }^{a, b, *}$ \\ ${ }^{a}$ CERN, Meyrin, Switzerland \\ ${ }^{b}$ AGH University of Science and Technology, Krakow, Poland \\ E-mail: Mateusz.Dyndal@cern.ch
}

Photon-photon fusion is a rare process at hadron and ion colliders. It is particularly interesting as a remarkably clean interaction with little (if any) remnant activity from the interacting particles. We present the status of photon-photon fusion measurements with the ATLAS detector at the LHC. This includes the production of lepton pairs and photon pairs (via light-by-light scattering) in heavy ion $(\mathrm{Pb}+\mathrm{Pb})$ collisions, as well as photon-photon fusion measurements in proton-proton collisions that contain two charged leptons in the final state. The experimental techniques used in the proton-proton and heavy-ion measurements are different, for example due to the different amounts of pile-up activity, and are also discussed.

40th International Conference on High Energy physics - ICHEP2020

July 28 - August 6, 2020

Prague, Czech Republic (virtual meeting)

\footnotetext{
${ }^{*}$ Speaker
} 


\section{Introduction}

Collisions of hadrons at high energies are usually studied for cases where the hadrons interact via the strong force, producing large particle multiplicities. The electromagnetic fields of the hadrons, however, can also induce interactions at large impact parameters where the strong interaction is not active (ultraperipheral collisions). In the 1930s it was found that the electromagnetic fields produced by the colliding charged particles can be treated as a beam of nearly-real photons emitted coherently [1]. This can give rise to photon-photon interactions, as schematically presented in Figure 1.

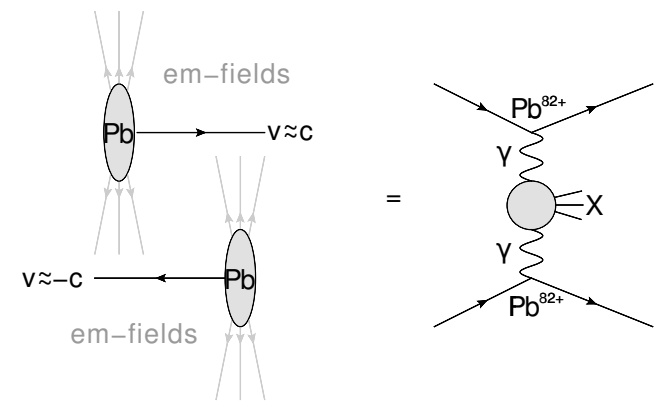

Figure 1: Illustration of an ultraperipheral collision of two lead ions [2]. Electromagnetic interaction between the ions can be described as an exchange of photons that can couple to form a given final-state $X$. The flux of photons is determined from the Fourier transform of the electromagnetic field of the ion, taking into account the nuclear electromagnetic form factors.

The nuclear cross section for a two-photon induced reaction can be calculated by convolving the respective photon flux with the elementary cross section for the $\gamma \gamma \rightarrow X$ subprocess. Since the photon flux associated with each hadron scales with its electric charge squared, the cross section in heavy ion $(\mathrm{Pb}+\mathrm{Pb})$ collisions at the LHC is extremely enhanced as compared to proton-proton $(p p)$ collisions. On the other hand, the $p p$ collisions provide a harder energy spectrum of initial photons, giving access to higher-energy phenomena (like the two-photon production of $W$ boson pairs).

The experimental signature of two-photon interactions is very striking: in the $2 \rightarrow 2$ exclusive processes the object pairs are typically produced without any other activity in the central detectors (exclusive production). The two objects are also produced back-to-back in azimuth, and have little total transverse momentum. This allows for good separation of signal and various background processes. However, in $p p$ collisions the pile-up (multiple interactions per bunch crossing) obscures the exclusivity of the photon-photon interactions and leads to an intrinsic complication in the reconstruction of exclusive final-states, which needs to be mitigated using dedicated experimental techniques.

The large $\mathrm{Pb}+\mathrm{Pb}$ and $p p$ datasets collected by the ATLAS experiment [3] during LHC Run 2 (2015-2018) allow for the precise study of various two-photon interaction processes.

\section{2. $\gamma \gamma \rightarrow \mu \mu$ production in $\mathrm{Pb}+\mathrm{Pb}$ collisions and forward neutron tagging}

ATLAS has measured the cross sections for exclusive dimuon production $(\gamma \gamma \rightarrow \mu \mu)$ in ultra-peripheral $\mathrm{Pb}+\mathrm{Pb}$ collisions for dimuon invariant masses $\left(m_{\mu \mu}\right)$ above $10 \mathrm{GeV}$ [4]. The 
cross sections are extracted by selecting events using a single-muon trigger, in association with an otherwise low charged particle multiplicity event. The events are required to have two oppositelycharged muons, each having transverse momentum $p_{\mathrm{T}}>4 \mathrm{GeV}$ and pseudorapidity $|\eta|<2.4$, with dimuon transverse momentum below $2 \mathrm{GeV}$. The events are then categorized with respect to energy deposits in Zero Degree Calorimeters (ZDC), which are sensitive to neutrons emitted as a result of $\mathrm{Pb}$ ion excitation due to multiple Coulomb interactions accompanying the $\gamma \gamma \rightarrow \mu \mu$ process. Three categories are defined ( $0 \mathrm{n} 0 \mathrm{n}, \mathrm{XnOn}$ and $\mathrm{XnXn}$ ) which reflect the ZDC energy activity on either side of the ATLAS detector. Here On denotes no neutron emission and Xn denotes any neutron emission.

The background, dominated by dissociative dimuon production where one photon is emitted by charged constituents of a nucleon, is estimated using template fits to dimuon acoplanarity (defined as $\alpha=1-\Delta \phi / \pi)$. This background is negligible for the 0n0n category, but it rises to $7 \%$ for $\mathrm{Xn} 0 \mathrm{n}$ and to $12 \%$ for $\mathrm{XnXn}$ categories.

The results are compared with calculations from the STARlight 2.0 MC generator [5], corrected for FSR effects using Pythia 8 [6], as presented in Figure 2. Generally, good agreement is found but some systematic differences are seen, which may be explained by deficiencies in the modeling of the incoming photon flux.
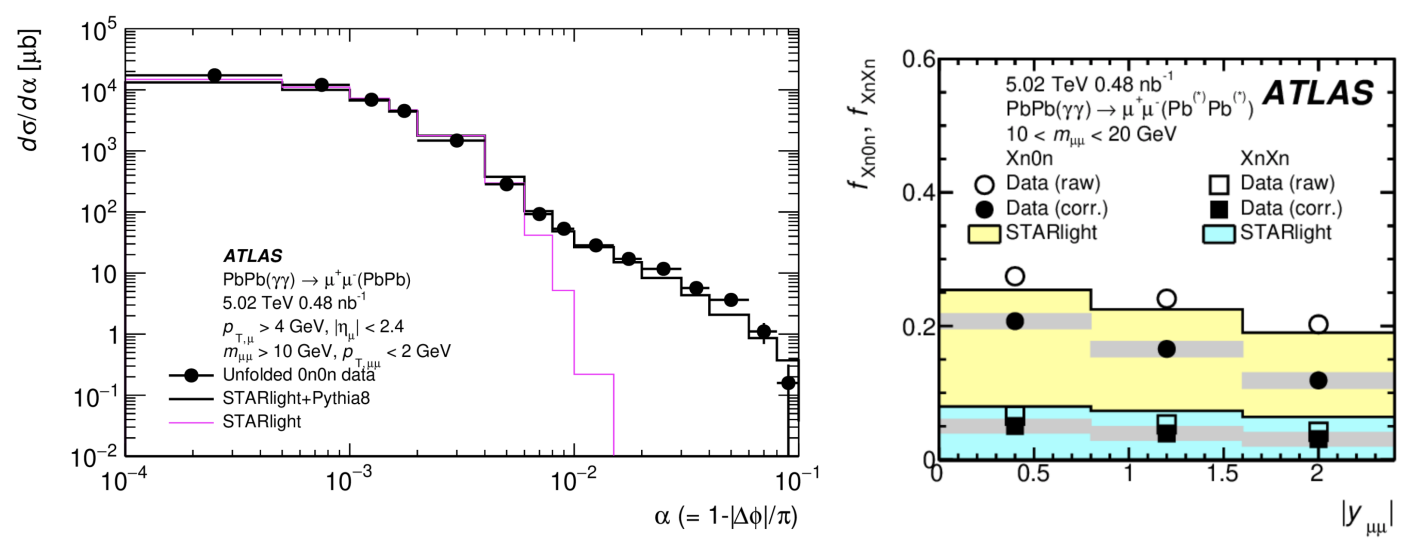

Figure 2: (left) Differential cross sections $\mathrm{d} \sigma / \mathrm{d} \alpha$ for $\gamma \gamma \rightarrow \mu \mu$ process in 0n0n-selection data [4]. Data are compared to absolute cross sections for STARlight with, and without, Pythia 8 QED showering. (right) Fractions of events with Xn0n and XnXn, as a function of dimuon rapidity for $10<m_{\mu \mu}<20 \mathrm{GeV}$ [4]. Data are shown as raw (open circles) and fully corrected for detector effects (closed circles).

\section{3. $\gamma \gamma \rightarrow \gamma \gamma$ scattering measurements in $\mathrm{Pb}+\mathrm{Pb}$ collisions and search for axion-like particles}

Following the observation of $\gamma \gamma \rightarrow \gamma \gamma$ (light-by-light scattering) process [7], a differential cross section measurement is performed by ATLAS using full Run-2 $\mathrm{Pb}+\mathrm{Pb}$ collision data [8]. Candidate diphoton events were recorded using a dedicated trigger for events with moderate activity in the calorimeter (covering the pseudorapidity range $|\eta|<4$.9) but little additional activity in the entire detector. Events are then required to have exactly two photons with transverse energy greater 
than $2.5 \mathrm{GeV}$, pseudorapidity $|\eta|<1.37$ or $1.52<|\eta|<2.37$, and a diphoton invariant mass greater than $5 \mathrm{GeV}$.

Background can arise mainly from misidentified electrons from the $\gamma \gamma \rightarrow e e$ process, as well as from the central exclusive production (CEP) of two photons from the fusion of two gluons (CEP $g g \rightarrow \gamma \gamma$ ). These backgrounds are estimated using data-driven methods.

Differential fiducial cross sections are measured as a function of several variables of the finalstate photons and are compared with standard model theory predictions [9]. Data and theory show fair agreement for all differential distributions, as demonstrated in Figure 3. The measurement precision is limited in all kinematic regions by statistical uncertainties.

The measured diphoton invariant mass distribution is used to search for axion-like particles (ALP) and set new exclusion limits on their production via the $\gamma \gamma \rightarrow a \rightarrow \gamma \gamma$ process. Integrated cross sections above 2 to $70 \mathrm{nb}$ are excluded at the $95 \% \mathrm{CL}$, depending on the diphoton invariant mass in the range $6-100 \mathrm{GeV}$. These results provide, to this date and within the aforementioned mass range, the most stringent constraints in the search for ALP signals (Figure 3).
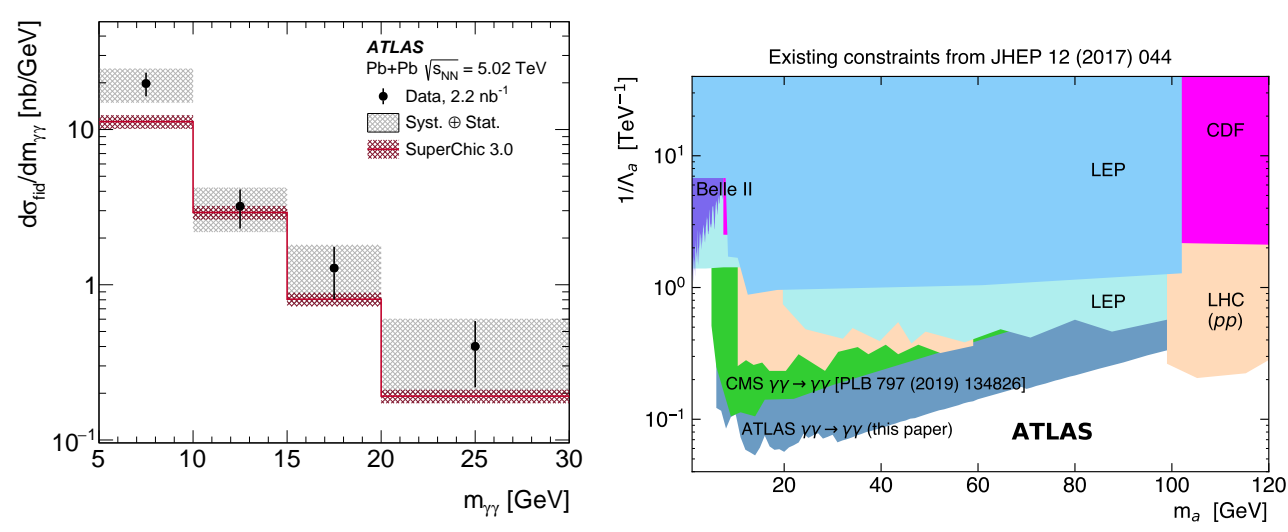

Figure 3: (left) Measured differential fiducial cross section of $\gamma \gamma \rightarrow \gamma \gamma$ production in $\mathrm{Pb}+\mathrm{Pb}$ collisions at $5.02 \mathrm{TeV}$ as a function of diphoton invariant mass [8]. The measured cross-section values are shown as points with error bars giving the statistical uncertainty and grey bands indicating the size of the total uncertainty. The results are compared with SuperChic 3 predictions (solid line) with bands denoting the theoretical uncertainty. (right) Compilation of exclusion limits at 95\% CL in the ALP-photon coupling $\left(1 / \Lambda_{a}\right)$ versus ALP mass $\left(m_{a}\right)$ plane obtained by different experiments [8]. The existing limits are compared with the limits extracted from this measurement.

\section{4. $\gamma \gamma \rightarrow \ell \ell$ production in $p p$ collisions with forward proton tag}

A measurement of proton-tagged dilepton production, $p p \rightarrow p(\gamma \gamma \rightarrow \ell \ell) p^{(*)}$, where $p^{(*)}$ denotes a proton that remains intact or dissociates following electromagnetic excitation, is performed [10]. The ATLAS Forward Proton (AFP) spectrometer [11] detects one of the intact protons and the central ATLAS detector reconstructs the leptons.

Several methods specific to proton-tagging are introduced: in situ calibration of proton kinematics using the dimuon system, a novel data-mixing background estimation method, and tag-and-probe determination of the AFP reconstruction efficiency. 
Signal event candidates having dilepton invariant mass $m_{\ell \ell}>20 \mathrm{GeV}$ and $m_{\ell \ell} \notin[70,105] \mathrm{GeV}$ are selected by having small dilepton acoplanarity and no inner-detector tracks around the dilepton vertex. This selection suppresses the region around the $Z$ boson mass, which is expected to be dominated by a Drell-Yan production. A pair of leptons from Drell-Yan process can mimic a signal event if detected in combination with a pile-up proton. The expected proton energy loss based on lepton kinematics, $\xi_{\ell \ell}$, is determined from $m_{\ell \ell}$ and the dilepton rapidity $y_{\ell \ell}$ by momentum conservation $\xi_{\ell \ell}^{ \pm}=\left(m_{\ell \ell} / \sqrt{s}\right) e^{ \pm y_{\ell \ell}}$. In order to suppress the background, $\xi_{\ell \ell}$ can be compared with the fractional energy loss of the scattered proton, $\xi_{\mathrm{AFP}}=1-E_{\text {scattered }} / E_{\text {beam }}$, where $E_{\text {scattered }}\left(E_{\text {beam }}\right)$ is the scattered (beam) proton energy (Figure 4 ). The scattered proton energy is obtained using the horizontal position of the proton reconstructed in AFP.

Cross-sections are measured in a fiducial region defined at particle-level with an event selection similar to that applied at detector level. The measurements are found to be in agreement with theoretical calculations that include soft-survival corrections.

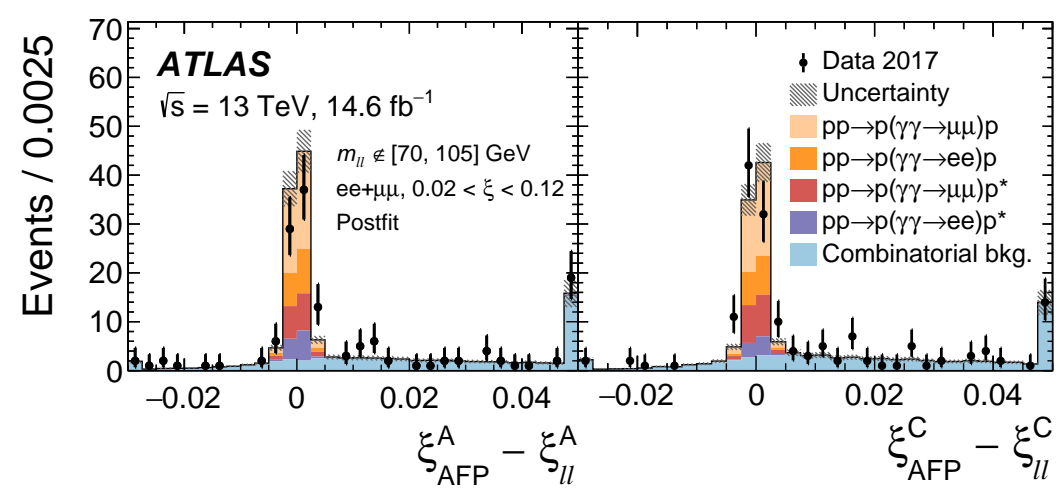

Figure 4: Distributions of $\xi_{\mathrm{AFP}}-\xi_{\ell \ell}$ with $\xi_{\ell \ell}$ and $\xi_{\mathrm{AFP}}$ in the range [0.02,0.12] for the two sides of the ATLAS detector [10]. The total prediction comprises the $\gamma \gamma \rightarrow \ell \ell$ signal and combinatorial background processes. The simulated predictions are normalized to data to illustrate the expected signal composition. The hatched band indicates the combined statistical and systematic uncertainties of the prediction. Error bars denote statistical uncertainties of the data.

\section{Observation of $\gamma \gamma \rightarrow W W$ scattering in $p p$ collisions}

The study of the $\gamma \gamma \rightarrow W W$ process using full Run-2 $13 \mathrm{TeV} p p$ dataset is presented by ATLAS [12]. This process is especially interesting due to the presence of a quartic $\gamma \gamma W W$ electroweak interaction vertex in the standard model.

The measurement follows the strategy established in previous ATLAS measurements [13, 14]. Events with leptonic $W$ boson decays into $e v_{e} \mu v_{\mu}$ final states were selected by requiring that no tracks except those of the two charged leptons are associated with the production vertex. Signal is extracted using a profile likelihood fit of the estimated signal and background event yields to data. The fit uses the integrated event yields in the signal region and three control regions.

Dedicated data-driven corrections are needed for this analysis to account for mismodelling of the additional $p p$ interactions produced in the same bunch crossing (pile-up), mismodelling of the 
charged-particle multiplicity in the inclusive $q q \rightarrow W W$ background process, and the dissociative contribution to the $\gamma \gamma \rightarrow W W$ signal process.

The background-only hypothesis is rejected with a significance of 8.4 standard deviations. The cross section for the sum of elastic and dissociative production mechanisms is measured and is in agreement with the theoretical predictions. This measurement constitutes the first observation of photon-induced $W W$ production in $p p$ collisions.

\section{Summary}

Recent ATLAS measurements of photon-photon fusion processes are presented. The measurements use $p p$ and $\mathrm{Pb}+\mathrm{Pb}$ collision data recorded in LHC Run 2. These new results include precision $\gamma \gamma \rightarrow \mu \mu$ measurements and updated $\gamma \gamma \rightarrow \gamma \gamma$ measurements in $\mathrm{Pb}+\mathrm{Pb}$ collisions, as well as observations of $\gamma \gamma \rightarrow \ell \ell$ production with forward proton tag and $\gamma \gamma \rightarrow W W$ scattering in $p p$ collisions. Such processes, as demonstrated for the $\gamma \gamma \rightarrow \gamma \gamma$ reaction, can be sensitive to specific beyond the standard model scenarios. All of this provides a novel and complementary research programme to test high-energy electroweak interactions.

This work was supported in part by Polish National Agency for Academic Exchange under the "Polish Returns 2020" Programme, Grant No. PPN/PPO/2020/1/00002/U/00001.

\section{References}

[1] E. J. Williams, Phys. Rev. 45 (1934), 729-730.

[2] ATLAS Collaboration, Nature Phys. 13 (2017) no.9, 852-858.

[3] ATLAS Collaboration, JINST 3 (2008), S08003.

[4] ATLAS Collaboration, arXiv:2011.12211 [nucl-ex].

[5] S. R. Klein, J. Nystrand, J. Seger, Y. Gorbunov and J. Butterworth, Comput. Phys. Commun. 212 (2017), 258-268.

[6] T. Sjöstrand et al., Comput. Phys. Commun. 191 (2015), 159-177.

[7] ATLAS Collaboration, Phys. Rev. Lett. 123 (2019) no.5, 052001.

[8] ATLAS Collaboration, arXiv:2008.05355 [hep-ex].

[9] L. A. Harland-Lang, V. A. Khoze and M. G. Ryskin, Eur. Phys. J. C 79 (2019) no.1, 39.

[10] ATLAS Collaboration, arXiv:2009.14537 [hep-ex].

[11] ATLAS Collaboration, ATLAS-TDR-024, http://cds.cern.ch/record/2017378.

[12] ATLAS Collaboration, arXiv:2010.04019 [hep-ex].

[13] ATLAS Collaboration, Phys. Rev. D 94 (2016) no.3, 032011.

[14] ATLAS Collaboration, Phys. Lett. B 777 (2018), 303-323. 